\title{
ESTUDO DO PROCESSO DE SECAGEM DA ENTRECASCA DE MELANCIA (Citrullus lanatus) PRODUZINDO A FARINHA UTILIZADA PARA CONFECÇÃO DE NOVOS PRODUTOS
}

\author{
Í. M. BACURAU ${ }^{1}$, F. V. AMORIM ${ }^{1}$, E. K. G. de OLIVEIRA ${ }^{1}$, V. C. FRANÇA ${ }^{1}$, M. M. de \\ ALMEIDA $^{2}$, M. R. LUIZ ${ }^{2}$ \\ ${ }^{1}$ Universidade Estadual da Paraíba, Departamento de Química \\ ${ }^{2}$ Universidade Estadual da Paraíba, Departamento de Engenharia Sanitária e Ambiental \\ E-mail para contato: marciarluiz@yahoo.com.br
}

\begin{abstract}
RESUMO - A melancia é altamente perecível e seu mesocarpo pode ser aproveitado para elaborar produtos em pó, dando um destino adequado a um resíduo que seria descartado. Uma das técnicas empregadas é a secagem convectiva. O objetivo deste trabalho foi estudar a cinética de secagem em diferentes temperaturas com variações de massa. O procedimento foi realizado a partir de um planejamento fatorial completo 23 com três repetições no ponto central. As variáveis independentes foram temperatura do ar de secagem e massa da amostra. A dependente foi a constante de secagem obtida a partir de modelos de secagem. A secagem foi desenvolvida sob as condições operacionais de massa de 100, 300 e $500 \mathrm{~g}$ e temperatura do ar de 50,60 e $70^{\circ} \mathrm{C}$. Foi possível observar, que a temperatura do ar de secagem teve maior influência em relação às variáveis independentes, como foi observado a partir da análise estatística e das curvas de cinéticas de secagem. O modelo que melhor representou os dados experimentais foi o de Page.
\end{abstract}

\section{INTRODUÇÃO}

O Brasil é um dos principais produtores de alimentos, porém enfrenta a realidade do desperdício em todas as etapas da cadeia produtiva. As perdas ocorrem nas centrais de abastecimento de todo o País e nos supermercados, sendo 30\% o total estimado. Segundo a Organização das Nações Unidas para Alimentação e Agricultura (FAO), o Brasil perde cerca de $60 \%$ de toda a produção anual de alimentos, o que onera o preço final, a quantidade e a qualidade dos produtos (FAO, 2008).

Uma forma de atuação, no que diz respeito ao aproveitamento de resíduos é a de buscar utilizações viáveis e econômicas para os inevitáveis resíduos agroindustriais gerados (FERNANDES, 2008). Neste sentido, o aproveitamento integral dos alimentos tem sido adotado como medida de fácil entendimento, pois é uma prática sustentável e ecologicamente correta, com maior utilização de recursos naturais que permite redução de gastos com alimentação da família, estimula a diversificação dos hábitos alimentares, bem como a questão nutricional com resíduos (SANTANA e OLIVEIRA, 2005) com a mesma carga nutricional que o popularmente consumido. 
Ressalta-se a importância dos talos, folhas, cascas e entrecascas que apresentam muitas vezes um aporte nutricional igual ou superior que da polpa. Os resíduos de frutas, leguminosas e hortaliças desprezados pelas indústrias e/ou de uso doméstico poderiam ser utilizados como fonte alternativa de nutrientes e de fibras alimentares (BOTELHO et al., 2002).

A entrecasca da melancia é um subproduto rico em fibra alimentar insolúvel. O seu aproveitamento na elaboração de produtos alimentícios pode contribuir para o aumento dos teores de fibra insolúvel na dieta, além de reduzir os desperdícios industriais (GUIMARÃES, 2007). Uma forma de aproveitamento para esses resíduos é a utilização de tecnologias relativamente baratas e de fácil acesso como os processos de secagem convectiva e de desidratação osmótica (PEREIRA et al., 2010).

Segundo Zen (2010), uma das técnicas de preservação de alimentos mais antigas usadas pelo homem é a de remoção de umidade dos alimentos pelo processo de secagem. E conforme Celestino (2010) este é o processo por meio do qual a água ou qualquer outro líquido é removido de um alimento sólido na forma de vapor por meio de um mecanismo de vaporização térmica com uma temperatura inferior a de ebulição da água.

Na secagem ocorrem dois processos simultâneos no material, a transferência de calor do meio circundante tendo como resultado a evaporação do líquido contido no material bem como, a transferência de massa na forma de líquido ou vapor do interior do material para a superfície, e desta para a corrente do fluido de secagem (COELHO, 2010).

O objetivo deste estudo é avaliar o processo de secagem do mesocarpo da melancia por meio do processo de secagem convectiva.

\subsection{Cinética de Secagem}

A secagem pode ser avaliada a partir de curvas, na forma de taxa de secagem que representam a forma mais simples de descrever a conduta da secagem de um material, em diferentes condições de operação dos secados e umidade inicial do produto (NOBREGA,2012).

As curvas de secagem são determinadas pela desidratação do material em condições pré-estabelecidas e pelo teor de umidade. Estas são alcançadas através da razão de umidade adimensional, como apresentado na Equação 1, em função do tempo de secagem. A razão de umidade é usada para impedir imprecisões dos resultados causados pelas diferenças no teor de umidade inicial da amostra (VELIC et al., 2004).

$$
X^{*}=\frac{X(t)-X e q}{X i-X e q}
$$

Onde: Xt: Teor de umidade a cada tempo de secagem; Xe: Teor de umidade de equilíbrio; Xi: Teor de umidade inicial. 


\subsection{Modelos Matemáticos}

Diversos modelos matemáticos são usados para descrever o processo de secagem e contribuir na sua otimização, auxiliando no planejamento eficaz de secadores. As equações matemáticas são utilizadas para representar a cinética de secagem e examinar o melhor ajuste aos dados experimentais (LUIZ, 2012). A Tabela 01 apresenta os modelos matemáticos mais utilizados para materiais agroindustriais e suas respectivas equações matemáticas.

Tabela 01 - Modelos matemáticos utilizados para descrever o fenômeno de secagem de materiais agroindustriais

\begin{tabular}{|c|c|}
\hline MODELO & EQUAÇÃO \\
\hline Midilli (2002) & $\mathrm{U}^{*}=\mathrm{a} . \exp (-\mathrm{k} . \mathrm{tn})+$ b.t \\
\hline Newton (RESENDE et al. (2009)) & $\mathrm{U}^{*}=\exp (-\mathrm{k} . \mathrm{t})$ \\
\hline Page (1949) & $\mathrm{U}^{*}=\exp (-\mathrm{k} . \mathrm{tn})$ \\
\hline
\end{tabular}

Onde: T: Tempo de secagem (min); k, n, a e b: Constantes dos modelos.

\section{METODOLOGIA}

As análises foram realizadas no Laboratório de Alimentos no Núcleo de Pesquisa e Extensão em Alimentos - NUPEA no Centro de Ciências e Tecnologia da Universidade Estadual da Paraíba. As entrecascas de melancias foram selecionadas de forma criteriosa visando garantir a homogeneidade em relação à coloração e aspecto físico e qualidade do produto final. Desprezaram-se os frutos que apresentaram danos físicos. As entrecascas foram lavadas em água corrente com detergente neutro e sanitizadas em solução de hipoclorito de sódio $100 \mathrm{ppm}$ por 15 minutos e cortadas para retirada total da polpa e da casca externa. A seleção das entrecascas foi realizada a partir do grau de maturação, tendo como referencial de 4,0 a $6,0^{\circ}$ Brix, medido com o auxílio de um refratômetro. Padronizou-se através dos experimentos a melhor espessura para a secagem. Os experimentos de secagem foram realizados nas temperaturas de 50,60 e $70^{\circ} \mathrm{C}$, em diferentes condições de secagem. Para verificação da umidade relativa do ar utilizou-se um termo higrômetro.

\subsection{Condições Operacionais e Metodologia Experimental}

Foi realizado o planejamento experimental para a secagem, onde se observam as etapas utilizadas no estudo da cinética de secagem em diferentes massas e condições de temperatura, respectivamente, como pode ser visto na Tabela 02.

Os experimentos de secagem foram realizados nas temperaturas de 50,60 e $70{ }^{\circ} \mathrm{C}$, com variação de peso nas amostras. A cinética de secagem foi estudada mediante as curvas do adimensional de umidade em função do tempo de processo, bem como pelas curvas de secagem em função do conteúdo de umidade das amostras. Os resultados experimentais das cinéticas de secagem foram usados para alimentar os modelos citados, buscando-se o ajuste que melhor represente os dados experimentais. 
Tabela 02 - Matriz do planejamento experimental $2^{3}$ com três repetições no ponto central

\begin{tabular}{|c|c|c|c|c|}
\hline ENSAIOS & $\mathbf{T}$ & $\mathbf{D}_{\mathbf{b}}$ & $\mathbf{T}\left({ }^{\mathbf{O}} \mathbf{C}\right)$ & $\mathbf{M}(\mathbf{g})$ \\
\hline 01 & -1 & -1 & 50 & 100 \\
\hline 02 & +1 & -1 & 70 & 100 \\
\hline 03 & -1 & +1 & 50 & 500 \\
\hline 04 & +1 & +1 & 70 & 500 \\
\hline 05 & 0 & 0 & 60 & 300 \\
\hline 06 & 0 & 0 & 60 & 300 \\
\hline 07 & 0 & 0 & 60 & 300 \\
\hline
\end{tabular}

\section{RESULTADOS E DISCUSSÕES}

\subsection{Cinética de secagem}

A secagem convectiva foi realizada em estufa com circulação de ar, a partir dos dados adimensionais de umidade definida como $\left.\mathrm{X}^{*}=\left[\mathrm{X}(\mathrm{t})-\mathrm{X}_{\mathrm{eq}}\right] /\left(\mathrm{X}_{\mathrm{i}}-\mathrm{X}_{\mathrm{eq}}\right)\right]$ e da taxa de secagem em função do tempo do processo. $\mathrm{O}$ estudo da cinética de secagem mostrou que o acréscimo da temperatura favoreceu a transferência de massa, diminuindo a umidade de equilíbrio dinâmico e o tempo de secagem.

Na Figura 01 estão apresentadas as curvas da cinética de secagem das amostras do mesocarpo da melancia que, geralmente, é descartado como rejeito.

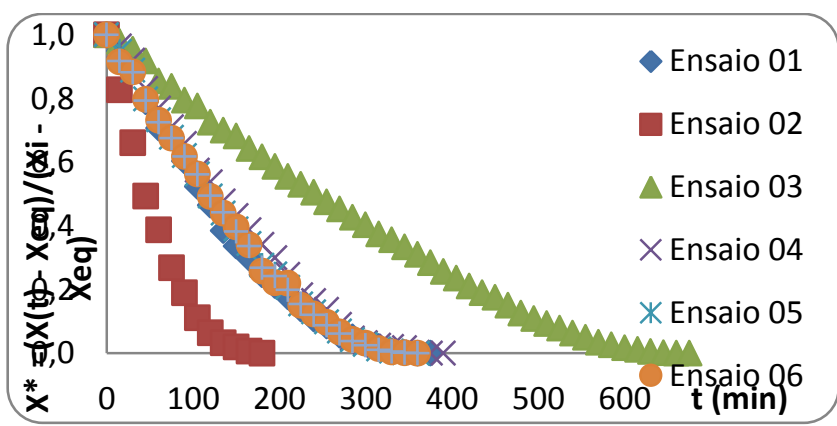

Figura 01 - Cinética de secagem de pedaços cúbicos do mesocarpo da melancia.

Verifica-se que para o ensaio 02, onde as condições operacionais são as de menor valor de temperatura do ar de secagem e menor massa do material a ser seco. Para o ensaio 03, em condições opostas ao ensaio 02 , observa-se que a secagem do mesocarpo da melancia ocorreu mais lentamente. Os ensaios 05, 06 e 07 representam os experimentos realizados nas condições de ponto central, desta maneira é possível observar uma boa reprodutibilidade dos dados experimentais obtidos, onde as curvas praticamente se sobrepõem umas sobre as outras. 
As Figuras 02 e 03 representam as curvas da variação da taxa de secagem em função da umidade média em base seca para os ensaios 02 e 03, respectivamente, o que configurou os outros ensaios.

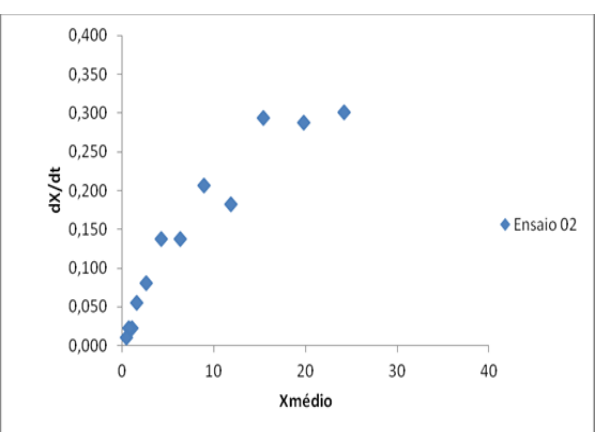

Figura 02 - Curva da taxa de secagem para o ensaio 02 .

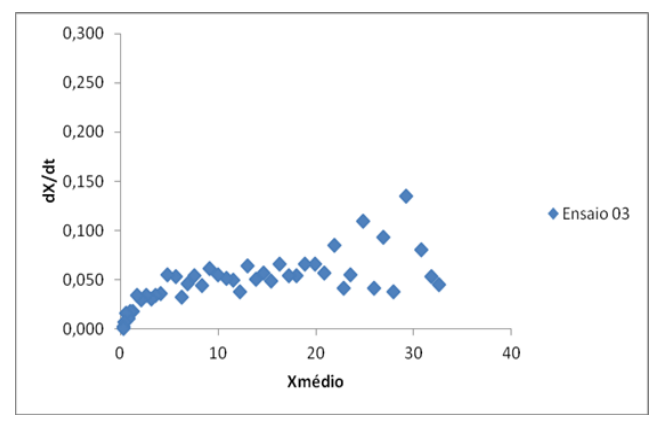

Figura 03 - Curva da taxa de secagem para o ensaio 03 .

Verifica-se que inicialmente a amostra passa por um rápido período de aquecimento com evaporação da água da superfície da mesma, obtendo-se altas taxas de secagem. Em seguida, observa-se nitidamente que a secagem ocorreu apenas em período de taxa decrescente.

Os modelos matemáticos descritos neste trabalho foram utilizados para representar os dados experimentais. Observou-se que o modelo de Page obteve melhor ajuste aos dados experimentais da secagem, como pode ser verificado nas Figuras 4, 5, 6, 7 e 8. Semelhante ao estudo de Azoubel et al. (2008), que durante a secagem de casca de manga 'Tommy Atkins' também verificaram que o melhor modelo para ajustar os dados experimentais da curva de secagem foi o modelo de Page.

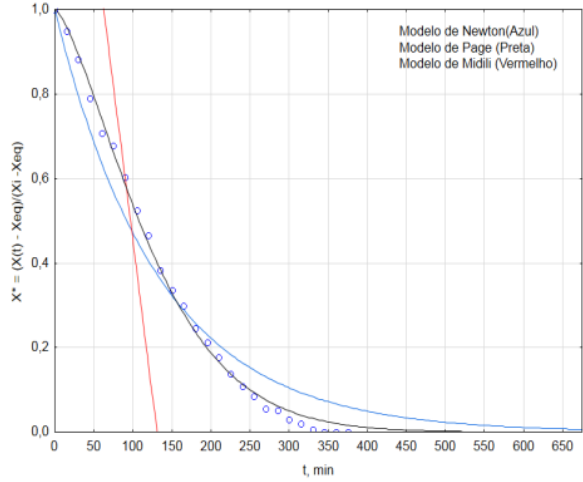

Figura 4 - Cinética de secagem representada pelos modelos matemáticos para o ensaio 01.

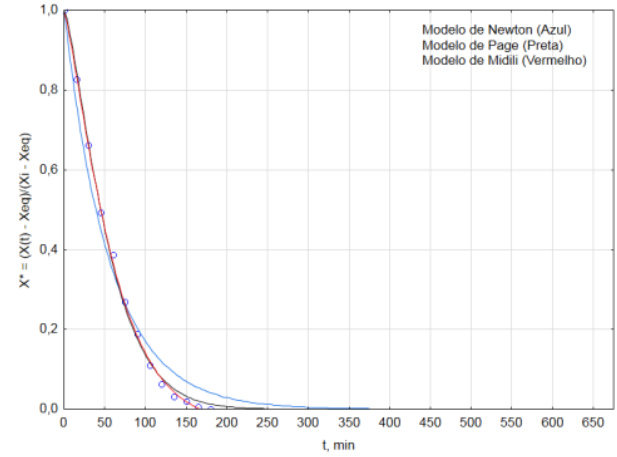

Figura 5 - Cinética de secagem representada pelos modelos matemáticos para o ensaio 02 . 


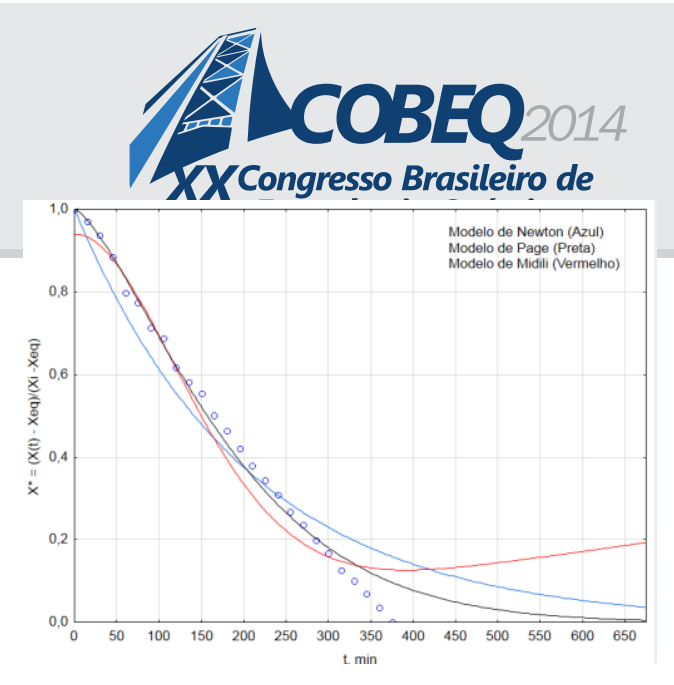

Figura 6 - Cinética de secagem representada pelos modelos matemáticos para o ensaio 03.
19 a 22 de outubro de 2014

Florianópolis/SC

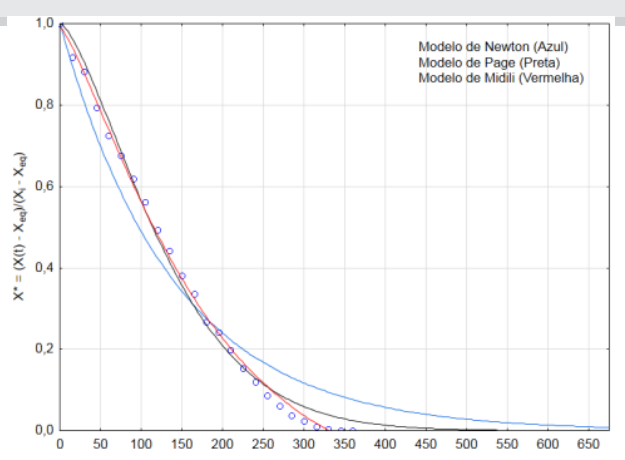

Figura 7 - Cinética de secagem representada pelos modelos matemáticos para o ensaio 04 .

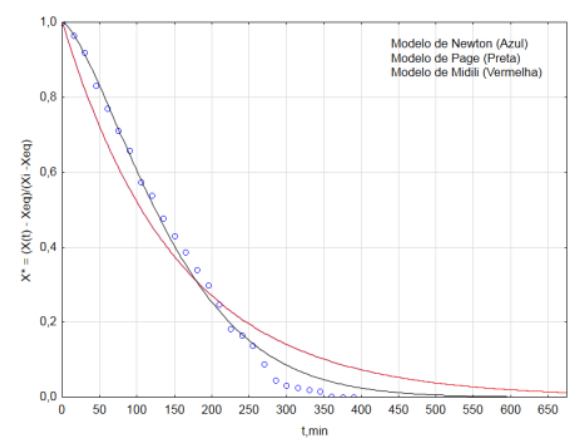

Figura 8 - Cinética de secagem representada pelos modelos matemáticos para o ensaio 05 .

A Tabela 03 apresenta os valores de $\mathrm{R}^{2}$, SE (erro padrão), AIC (método de Akaike) e os valores encontrados para os parâmetros dos modelos utilizados.

Tabela 03 - Resultados dos parâmetros dos modelos empíricos e análise estatística.

\begin{tabular}{|c|c|c|c|c|c|c|c|c|}
\hline \multirow{2}{*}{ MODELO } & \multirow{2}{*}{ ENSAIO } & \multicolumn{4}{|c|}{ PARÂMETROS } & \multirow{2}{*}{$\mathbf{R}^{2}$} & \multirow{2}{*}{$\mathbf{S E}$} & \multirow[t]{2}{*}{ AIC } \\
\hline & & A & $\mathbf{k}$ & $\mathbf{N}$ & b & & & \\
\hline \multirow{5}{*}{ Midilli } & 01 & 7,1927 & 1,3196 & 0,000008 & $-0,0176$ & & 2,234 & 47,717 \\
\hline & 02 & 0,9939 & 0,0054 & 1,2639 & $-0,00018$ & 0,9990 & 0,0106 & $-112,3$ \\
\hline & 03 & 1,4205 & 0,0702 & 0,000001 & 0,00404 & & & \\
\hline & 04 & 1,0217 & 0,0016 & 1,2388 & $-0,00028$ & 0,9964 & 0,0782 & $-131,7$ \\
\hline & 05 & 0,9914 & 0,0014 & 1,2777 & $-0,00027$ & 0,9978 & 0,0158 & $-201,6$ \\
\hline \multirow{5}{*}{ Newton } & 01 & - & 0,00753 & - & - & 0,9621 & 0,0653 & $-141,9$ \\
\hline & 02 & _- & 0,0177 & - & _ & 0,9795 & 0,0506 & $-77,77$ \\
\hline & 03 & - & 0,00345 & - & _- & 0,9498 & 0,0727 & $-242,5$ \\
\hline & 04 & $=$ & 0,0065 & & $=$ & 0,9457 & 0,0782 & $-137,7$ \\
\hline & 05 & - & 0,0071 & & _- & 0,9541 & 0,0717 & $-131,9$ \\
\hline \multirow{5}{*}{ Page } & 01 & _- & 0,0008 & 1,4328 & _ & 0,9972 & 0,0179 & $-207,4$ \\
\hline & 02 & $\ldots$ & 0,0045 & 1,3250 & - & 0,9978 & 0,0161 & $-105,6$ \\
\hline & 03 & _ & 0,0004 & 1,3755 & _ & 0,9892 & 0,0331 & $-311,6$ \\
\hline & 04 & _- & 0,0006 & 1,4402 & _ & 0,9926 & 0,0286 & $-189,9$ \\
\hline & 05 & & 0,0007 & 1,4547 & & 0,9932 & 0,0277 & $-177,4$ \\
\hline
\end{tabular}

Onde: $\mathrm{R}^{2}=$ coeficiente de determinação; $\mathrm{SE}=$ erro padrão; $\mathrm{AIC}=$ método de Akaike; $\mathrm{a}, \mathrm{k}$, n e $\mathrm{b}=$ parâmetros do modelo. 


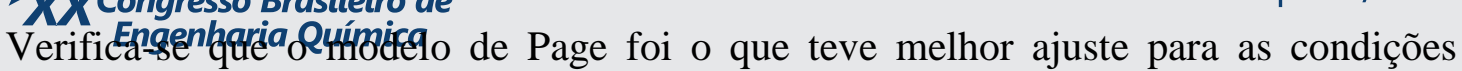
operacionais utilizadas no planejamento experimental estudado, pois os valores do erro padrão (SE) são menores, enquanto os outros modelos, apesar de apresentarem valores do coeficiente de determinação $\left(\mathrm{R}^{2}\right)$ próximos do valor um. Os valores do erro padrão encontrados foram superiores aos sugeridos por Lomauro et al. (1985). Resultados similares foram encontrados por El-Aouar et al. (2003) para o mamão, enquanto Doymaz (2004) observou que os modelos Exponencial de dois termos e o de Wang e Singh foram os que melhor representaram os dados experimentais da secagem de ameixa. Também, pode-se verificar estatisticamente pelo método de Akaike que o modelo de Page ajusta melhor os dados experimentais com valores mais negativos deste método.

\subsection{Análise estatística dos resultados}

Nas Figuras 9 e 10 estão ilustrados os resultados dos valores observados e dos valores preditos pelos modelos, de acordo com cada variável de resposta.

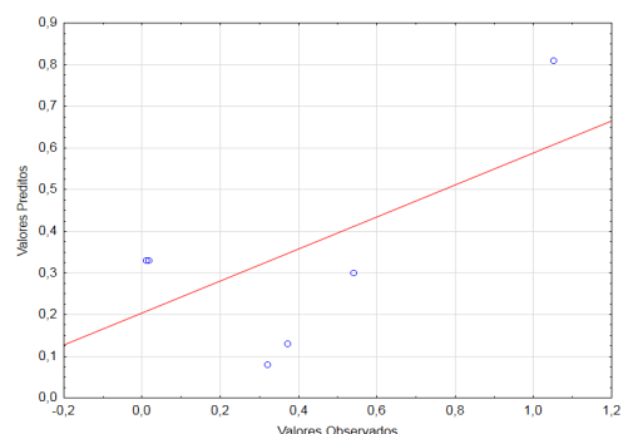

Figura 9 - Valores observados versus valores preditos para variável resposta $\mathrm{X}_{\mathrm{f}}$.

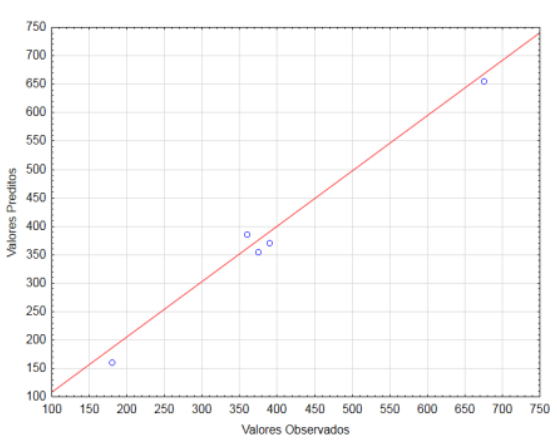

Figura 10 - Valores observados versus e preditos para variável resposta $t_{\text {secagem. }}$.

De acordo com a figura 9, verifica-se que para os valores de umidade final do produto em base seca (Xf) em relação aos valores preditos observa-se uma grande dispersão, desta forma pode-se afirmar que o modelo supostamente linear previsto estatisticamente apresenta uma má qualidade do ajuste. Em relação ao tempo de secagem (tsecagem), representado na Figura 10, os valores apresentaram uma boa concordância.

As Figuras 11 e 12 representam os diagramas de Pareto para as variáveis respostas umidade final do produto em base seca e tempo de secagem.

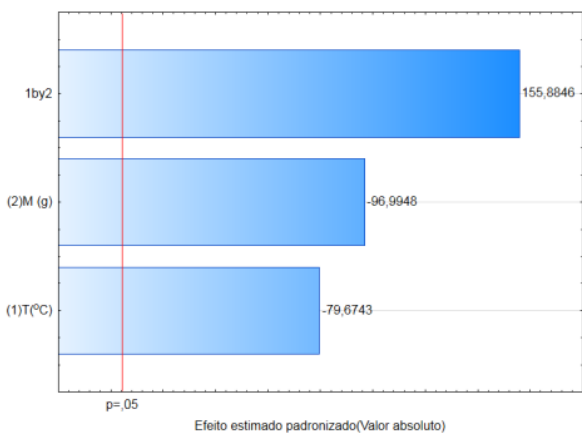

Figura 11 - Diagrama de Pareto para variável resposta $X_{\mathrm{f}}$.

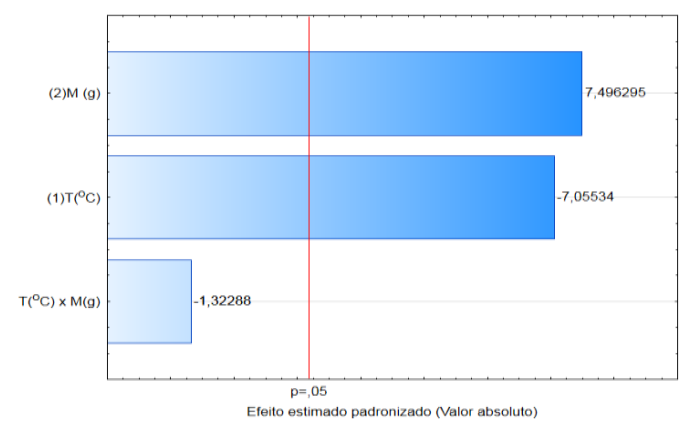

Figura 12 - Diagrama de Pareto para variável resposta $t_{\text {secagem. }}$. 


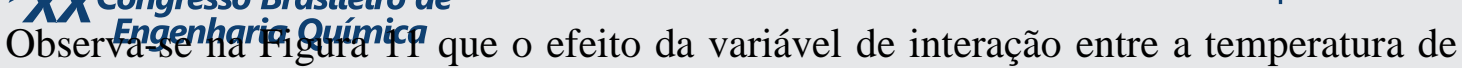
secagem e a massa inicial do produto apresenta um efeito positivo, sendo este mais significativo. Por outro lado, Os efeitos da massa inicial do produto e da temperatura apresentam um efeito negativo, ou seja, quanto maior este valor menor a resposta analisada.

$\mathrm{Na}$ Tabela 04 estão apresentados os valores de comparação do coeficiente de determinação para os modelos linear e de curvatura.

Tabela 04 - Comparação do coeficiente de determinação para o modelo linear e modelo com curvatura estatístico.

\begin{tabular}{|l|c|c|c|c|}
\hline \multirow{2}{*}{$\begin{array}{l}\text { COEFICIENTE DE } \\
\text { DETERMINAÇÃO }\end{array}$} & \multicolumn{2}{|c|}{ Xf (base seca) } & \multicolumn{2}{c|}{ T$_{\text {Secagem }}$ (min) } \\
\cline { 2 - 5 } & Linear & Curvatura & Linear & Curvatura \\
\hline $\mathrm{R}^{2}$ & 0,3844 & 0,9729 & 0,9999 & 1,000 \\
\hline
\end{tabular}

\section{CONCLUSÕES}

De acordo com os resultados obtidos, percebe-se que a cinética da secagem convectiva apresentou um ótimo resultado em relação à temperatura de secagem. As respostas obtidas com os ensaios dos pontos centrais mostrou boa reprodutibilidade dos dados experimentais.

Entre as variáveis operacionais estudadas os resultados preliminares mostraram que o efeito da variável de interação entre a temperatura de secagem e a massa inicial do produto apresentou um efeito positivo, sendo este mais significativo. Por outro lado, os efeitos da massa inicial do produto e da temperatura apresentaram um efeito negativo, ou seja, quanto maior este valor menor a resposta analisada. Observou-se que com o aumento da temperatura e a diminuição da massa os resultados foram mais satisfatórios, havendo uma redução na umidade final do produto obtido.

Portanto, pode-se considerar, de uma maneira geral, que este estudo de secagem convectiva com o mesocarpo da melancia (Citrullus lanatus) com o planejamento $2^{3}$ com três pontos centrais, apresentou resultados bastante satisfatórios para o modelo de Page. Sendo assim, considerado um processo viável para utilização na fabricação de um produto que pode ser comercializado, minimizando o desperdício de resíduos agroindustriais.

\section{REFERÊNCIAS}

AZOUBEL, P. M.; EVANGElistA, E. C. D. A.; DE OliveIRA, S.B.; SILVA, Í. R. A.; ARAÚJO, A. J. B. Cinética de secagem da casca de manga 'tommy atkins'. XVII Congresso Brasileiro de Engenharia Química, Recife. 2008.

CELESTinO, S. M. C. Princípios de Secagem de Alimentos. Planaltina, DF: Embrapa Cerrados, 2010.

COELHO, K. D. Secagem de tomate (lycorpesicon esculentum mill) em camada delgada: Avaliação das características físico-químicas. Dissertação (Mestrado em Engenharia e Ciências de Alimentos, Programa de Pós-Graduação em Engenharia e Ciências de Alimentos) - Universidade Federal do Rio Grande, Rio Grande, 2010.

DOYMAZ, I. Effect of dipping treatment on air drying of plums, J. of Food Eng., v. 64, p. 465-470, 2004. 
EL-AOUAR, ERg.qnharizQứBieq, P. M.; MURR, F. E. X. Drying kinetics of fresh and osmotically pretreated papaya (Carica papaya L.), J. of Food Eng., v. 59, p. 85-91, 2003.

FAO - Organizacao das Nacoes Unidas para Alimentacao e Agricultura. Corporate DocumentRepository. Crop Prospects and Food Situation - No. 4, 2008. Disponivel em:〈www.fao.org>. Acesso em: 20 dez. 2008.

FERNANDES, A. F. Efeito da substituição parcial da farinha de trigo por farinha de casca de batata (SolanumTuberosum Lineu). Ciênc. Tecnol. Aliment., 2008.

GUIMARÃES, R. R. Avaliação nutricional da farinha da entrecasca de melancia (Citrullusvulgaris Sobral) em animais. In: Simpósio Latino Americano de Ciência de Alimentos, 2007.

LOMAURO, C. J.; BAKSHI, A. S.; LABUZA, T. P.Evaluation of food moisture sorption isotherm equations. Part I: fruit, vegetable and meat products. Lebens.-Wiss. und Tech., v. 18, p. 112-122, 1985.

LUIZ, M. R. Estudo teórico e experimental de secagem de tomate. Tese (Doutorado) Universidade Federal da Paraíba, UFPB, João Pessoa, 2012.

MIDILLI, A.; KUCUK, H.; YAPAR, Z. A. New model for single-layer drying. Drying Technology, v.20, p.1503-1513, 2002.

NÓBREGA, E. M. M. de. Secagem do resíduo de acerola (Malphigiaemarginata D. C.): Estudo do processo e avaliação do impacto sobre o produto final. Dissertação (Mestrado em Engenharia Química, Programa de Pós-Graduação em Engenharia Química) - Universidade Federal do Rio Grande do Norte, UFRN, Natal, 2012.

PAGE, G. E. Factors influencing the maximum of air drying shelled corn in thin layer. Dissertação de mestrado. Purdue University, Indiana, USA, 1949.

PEREIRA, A. S., MiguEl, D. P., CARVAlho E. E. N. Caracterização de Farinha da Entrecasca de Melancia (citrulluslanatus) Produzida na Região Sul do Tocantins. Cadernos de Pós-graduação da FAZU, V. 1, 2010.

SANTANA, A. F.; OLIVEIRA, L. F. Aproveitamento da casca de melancia (Curcubitacitrullus, Shrad) na produção artesanal de doces alternativos. Alim. Nutr., Araraquara, v. 16, n.4, p. 363-368, out./dez. 2005.

VELIC, D.; PLANINIC, M. TOMAS, S. BILIC, M. Influence of airflow velocity on kinetics of convection apple drying. Journalof Food Engineering, 64, p. 97-102, 2004. 